\title{
Comparative Evaluation of Voice Activity Detectors in Single Microphone Noise Reduction Algorithms
}

\author{
Jonghee Han, Sunhyun Yook, Kyoung Won Nam, Sangmin Lee, Dongwook Kim, Sung Hwa Hong, Dong Pyo Jang, \\ and In Young Kim
}

Received: 27 November 2012 / Accepted: 20 December 2012

(C) The Korean Society of Medical \& Biological Engineering and Springer 2012

In the 2012 issue of Medical \& Biological Engineering, an error occurred in the research article:

Jonghee Han, Sunhyun Yook, Kyoung Won Nam, Sangmin Lee, Dongwook Kim, Sung Hwa Hong, Dong Pyo Jang, and In Young Kim (2012)

Comparative Evaluation of Voice Activity Detectors in Single Microphone Noise Reduction Algorithms.

Biomed Eng Lett. (2012) 2:255-264

\section{- In the affiliation}

\section{Original wording:}

Jonghee Han, Dongwook Kim, In Young Kim

Samsung Advanced Institute of Technology, P.O. Box 111, Suwon, 440-600, Korea

Sunhyun Yook, Kyoung Won Nam, Dong Pyo Jang Department of Biomedical Engineering, Hanyang University, Seongdong P.O. Box 55, Seoul, 133-791, Korea

Jonghee Han, Sunhyun Yook, Kyoung Won Nam, Dong Pyo Jang, In Young Kim

Department of Biomedical Engineering, Hanyang University, Seongdong P.O. Box 55, Seoul, 133-791, Korea

Dongwook Kim

Samsung Advanced Institute of Technology, P.O. Box 111, Suwon, 440600 , Korea

Sangmin Lee

Department of Electronic Engineering, Inha University, Incheon, 402-751, Korea

Sung Hwa Hong

Department of ORL HNS, Samsung Medical Center, Sungkyunkwan University, School of Medicine, Korea

In Young $\operatorname{Kim}(\square)$

Fusion Technology Center, Hanyang University, Seongdong P.O. Box 55, Seoul, 133-791, Korea

Tel : +82-2-2291-1713 / Fax : +82-2-2220-4949

E-mail : iykim@hanyang.ac.kr
Sangmin Lee

Department of Electronic Engineering, Inha University, Incheon, 402-751, Korea

Sung Hwa Hong

Department of ORL_HNS, Samsung Medical Center, Sungkyunkwan University, School of Medicine, Korea

In Young Kim $(\varangle)$

Fusion Technology Center, Hanyang University, Seongdong P.O. Box 55, Seoul, 133-791, Korea

Tel : +82-2-2291-1713 / Fax : +82-2-2220-4949

E-mail : iykim@hanyang.ac.kr

This should be replaced by:

Jonghee Han, Sunhyun Yook, Kyoung Won Nam, Dong Pyo Jang, In Young Kim

Department of Biomedical Engineering, Hanyang University, Seongdong P.O. Box 55, Seoul, 133-791, Korea

Dongwook Kim

Samsung Advanced Institute of Technology, P.O. Box 111, Suwon, 440-600, Korea

Sangmin Lee

Department of Electronic Engineering, Inha University, Incheon, 402-751, Korea

Sung Hwa Hong

Department of ORL_HNS, Samsung Medical Center, Sungkyunkwan University, School of Medicine, Korea

In Young Kim $(\bowtie)$

Fusion Technology Center, Hanyang University, Seongdong P.O. Box 55, Seoul, 133-791, Korea

Tel : +82-2-2291-1713 / Fax : +82-2-2220-4949

E-mail : iykim@hanyang.ac.kr 